\title{
TRANSTORNO MENTAL E ESTRESSORES NO TRABALHO ENTRE PROFESSORES UNIVERSITÁRIOS DA ÁREA DA SAÚDE
}

MENTAL DISORDER AND STRESS FACTORS AT WORK AMONG HEALTH AREA PROFESSORS

\author{
TRASTORNO MENTAL Y ESTRESORES EN EL TRABAJO ENTRE PROFESORES UNIVERSITARIOS \\ EN EL ÁREA DE LA SALUD
}

\author{
Raquel Conceição Ferreira ${ }^{1}$ \\ Alessandra Pastore da Silveira ${ }^{2}$ \\ Maria Aparecida Barbosa de Sá ${ }^{3}$ \\ Sara de Barros Lima Feres ${ }^{4}$ \\ João Gabriel Silva Souza ${ }^{5}$ \\ Andréa Maria Eleutério de Barros Lima Martins ${ }^{6}$
}

Resumo Os professores universitários estão expostos a um aumento de tensão no trabalho pela fragmentação da sua atividade e as responsabilidades exigidas, sem que, em muitas situações, tenham as condições necessárias para responder adequadamente. Tal situação pode representar condições estressoras, aumentando o risco de transtornos mentais. Investigou-se a associação entre transtornos mentais comuns e estressores no trabalho entre professores de nove cursos da área da saúde de uma universidade particular em Minas Gerais. A variável dependente foi a presença de transtornos mentais, avaliada pelo Questionário de Saúde Geral 12. Os estressores no trabalho foram avaliados pelo modelo Esforço-Recompensa e Comprometimento excessivo. As demais variáveis foram: sociodemográficas, história ocupacional, comportamentais e referentes à saúde geral. Os dados foram submetidos à análise descritiva, análise bivariada e regressão de Poisson. Participaram 175 professores (80,0\%), e 19,5\% apresentaram transtornos mentais comuns. A prevalência desses transtornos foi maior entre professores com maior esforço no trabalho $(\mathrm{RP}=1,8$; IC95\% $=1,01-3,46)$ e menor naqueles com maior qualidade de vida no domínio físico $(\mathrm{RP}=0,95$, IC95\%=0,93-0,97). Conclui-se que há uma prevalência considerável de transtornos mentais comuns entre professores universitários, sendo maior naqueles que se esforçam mais no trabalho e com pior qualidade de vida no domínio físico.

Palavras-chave saúde mental; docentes; qualidade de vida; saúde do trabalhador; esgotamento profissional.
Abstract Professors are exposed to increased tension at work on account of the fragmentation of their activities and of the responsibilities demanded of them without, in many cases, their having the conditions they need in order to respond adequately. Such a situation may represent stressful conditions, which increase the risk of mental disorders. We investigated the association between common mental disorders and stress factors at work among professors teaching in nine health courses at a private university in Minas Gerais, Brazil. The dependent variable was the presence of mental disorders, as measured by General Health Questionnaire 12. Stress factors at work were evaluated based on the Effort-Reward and Excessive Commitment model. The other variables were sociodemographic, occupational background, behavioral, and related to general health. The data were submitted to descriptive analyses, bivariate analyses, and Poisson regression. A total of 175 professors participated $(80.0 \%)$, of whom $19.5 \%$ had mental disorders. These disorders were more prevalent among professors making the most effort at work $(\mathrm{PR}=1.8,95 \% \mathrm{CI}=1.01$ 3.46) and less prevalent among those with higher quality of life in their physical domain $(\mathrm{PR}=0.95$, $95 \% \mathrm{CI}=0.93$ to 0.97 ). It can be concluded that there is a considerable prevalence of common mental disorders among university professors, which were higher among those who put more effort into their work and had lower quality of life in their physical domains.

Keywords mental health; professors; quality of life; worker's health; burnout. 


\section{Introdução}

As interações estabelecidas entre os trabalhadores e as suas condições de trabalho podem comprometer a saúde. As transformações técnicas e organizacionais do trabalho têm gerado significativas consequências para a vida e a saúde dos trabalhadores em geral, o que condiciona mudanças no seu perfil de morbimortalidade, com aumento de doenças mentais, psicossomáticas, cardiovasculares e osteoarticulares, entre outras (Gasparini, Barreto e Assunção, 2006).

Transtornos mentais são condições caracterizadas por alterações mórbidas do modo de pensar e do humor (emoções), ou por alterações mórbidas do comportamento, associadas à angústia expressiva e deterioração do funcionamento psíquico global (Delcor et al., 2004). Jacques (2007) relatou que, segundo estimativas da Organização Mundial de Saúde (OMS), os transtornos mentais menores acometem cerca de 30\% dos trabalhadores ocupados e os transtornos mentais graves, cerca de $5 \%$ a $10 \%$. No Brasil, segundo estatísticas do Instituto Nacional do Seguro Social, referentes apenas aos trabalhadores com registro formal, os transtornos mentais ocupam a terceira posição entre as causas de concessão de benefício previdenciário como auxílio doença, afastamento do trabalho por mais de 15 dias e aposentadorias por invalidez (Brasil, 2001).

Os transtornos mentais afetam negativamente a satisfação com a vida e a qualidade de vida e, por serem incapacitantes, representam um alto custo social e econômico, pois levam a dias perdidos de trabalho e aumento na demanda por serviços de saúde (OMS, 2001; Ludermir e Filho, 2002; Gonçalves e Kapczinski, 2008; Brum et al., 2012).

Os profissionais envolvidos em atividades em que há o contato direto com o público, como os da educação e da saúde, estão mais expostos aos riscos psicossomáticos (Gasparini, Barreto e Assunção, 2006; Carlotto e Palazzo, 2006). Entre os docentes, os transtornos mentais situam-se entre as principais queixas de saúde (Delcor et al., 2004). A incorporação de novas tecnologias, as mudanças no sistema educacional e nas formas de organização do trabalho, com cargas de trabalho fatigantes, representam transformações no cotidiano dos professores. Essa situação coloca o professor em condição de maior vulnerabilidade ao sofrimento e ao adoecimento (Fernandes e Rocha, 2009; Fontana e Pinheiro, 2010).

A nova configuração do trabalho do professor universitário pode conduzir a situações de enriquecimento e satisfação, mas pode também ocasionar ou incrementar fontes de estresse (Carlotto, 2010). Estresse e insatisfação são conceitos inter-relacionados, significando que estressores no trabalho contribuem para a insatisfação, que ajudam a aumentar os níveis de estresse (Abouserie, 1996). 
Os professores não têm apenas de ensinar, realizar certificados de exames, avaliar trabalhos e escrever artigos, mas também devem submeter seus projetos a financiamentos e ter seus trabalhos publicados (Leung, Siu e Spector, 2000). Além das atividades citadas, precisam ainda: participar de reuniões, comissões, atender e orientar alunos, planejar aulas, apresentar trabalhos, fazer relatórios, relatar processos, cumprir prazos, entre outras. Não raro, falta tempo ao professor para cumprir suas múltiplas responsabilidades. Segundo Carlotto (2010),

professores sofrem as consequências de estarem expostos a um aumento de tensão no exercício de seu trabalho, cuja dificuldade aumentou, fundamentalmente pela fragmentação da atividade do professor e o aumento de responsabilidades que lhe são exigidas, sem que, em muitas situações, tenham os meios e condições necessários para responder adequadamente (Carlotto, 2010, p. 189).

Os professores universitários foram o grupo de trabalhadores considerados por pesquisadores em estudos de saúde ocupacional (Abouserie, 1996; Gillespie et al., 2001; Sousa e Mendonça, 2009; Carlotto, 2010; Mark e Smith, 2012). Abouserie (1996) observou que 74\% dos professores universitários da Universidade de Wales, no Reino Unido, mencionaram o trabalho como a principal fonte de estresse em suas vidas e, no trabalho, a realização de pesquisas $(40,3 \%$ ) foi a principal fonte de estresse citada. Profissionais acadêmicos, de universidades australianas, relataram como cinco principais fontes de estresse: financiamento e recursos insuficientes, sobrecarga de trabalho, má gestão, insegurança no emprego, reconhecimento e recompensa insuficientes (Gillespie et al., 2001).

Dois estudos nacionais investigaram a síndrome de burnout entre professores universitários, definida como uma reação a fontes de estresse ocupacional contínuas que se acumulam (Sousa e Mendonça, 2009; Carlotto, 2010). Entre docentes de uma universidade privada da região Centro-Oeste, observou-se que a percepção de injustiça na forma de distribuição de recursos pode levar o professor à exaustão emocional, o que pode ter probabilidade aumentada diante da falta de comprometimento do professor (Sousa e Mendonça, 2009). Em outro estudo nacional, os níveis de síndrome de burnout foram avaliados entre professores de uma universidade particular que exerciam suas funções em atividade de ensino, pesquisa e extensão. Nesse estudo, verificou-se que os professores locados nos Centros da Saúde e no Centro de Ciências Humanas apresentaram maiores níveis de burnout, principalmente quanto à exaustão emocional, quando comparados com os colegas dos centros de Ciências Exatas e do Centro de Ciências Jurídicas (Carlotto, 2010).

A associação entre estressores e transtornos mentais foi investigada entre trabalhadores de uma universidade no Reino Unido, que apresentaram maior frequência de ansiedade e depressão comparativamente à população 
em geral. Demandas, esforço e comprometimento excessivo no trabalho, bem como baixa resiliência foram associados com alto nível de depressão, ansiedade e baixa satisfação com o trabalho (Mark e Smith, 2012).

No Brasil, os últimos anos foram marcados pela expansão do ensino universitário em instituições privadas, acompanhada de uma mudança no papel do professor na tentativa de atender às expectativas e necessidades da sociedade atual, que supõe uma ampla série de habilidades que não podem ser reduzidas ao âmbito do acúmulo de conhecimento (Carlotto, 2010).

Desse modo, há necessidade de novos estudos em contextos específicos, que contribuam para a compreensão dos efeitos de estressores do trabalho na saúde dos professores. O objetivo deste estudo foi avaliar a associação entre estressores no trabalho e transtornos mentais comuns entre docentes da área da saúde, no contexto de uma universidade particular.

\section{Percurso metodológico}

Tratou-se de um estudo transversal entre todos os professores dos nove cursos da área da saúde de uma instituição privada de ensino superior do norte de Minas Gerais: Medicina, Biomedicina, Odontologia, Enfermagem, Farmácia, Educação Física, Fonoaudiologia, Fisioterapia e Nutrição. A lista de professores foi fornecida pelo setor de recursos humanos da universidade. Na época da coleta de dados, 224 professores trabalhavam na instituição, uma professora estava de licença maternidade e dois estavam de licença médica, totalizando 221 professores elegíveis. Desses 221, 177 (80\%) responderam os questionários. Dos 44 (20\%) professores não respondentes, 29 recusaram-se a participar e 15 não foram encontrados após três agendamentos. A maioria dos não respondentes era professor do curso de Medicina (68,2\%) e 55,0\% eram do sexo feminino. No estudo, foram incluídos 175 professores, pois outros dois foram eliminados por terem respondido menos de $20,0 \%$ das questões do instrumento usado para avaliação de qualidade de vida, o World Health Organization Quality of Life (WHOQOL-bref), conforme orientações da OMS para sua análise.

Os dados foram coletados por meio de um questionário autoaplicável, composto por questões elaboradas pelos pesquisadores (avaliação de características sociodemográficas, história ocupacional, variáveis comportamentais e referentes a saúde geral) e de instrumentos validados no Brasil (Questionário de Saúde Geral - QSG-12; Escala de Esforço, Recompensa e Comprometimento excessivo no trabalho; Questionário Internacional de atividades Físicas -IPAQ; WHOQOL-bref). O questionário foi testado em um grupo de dez professores universitários que não trabalhavam na instituição investigada, a fim de avaliar a sequência das perguntas, tempo gasto para resposta e necessidade de adequações nas questões elaboradas pelos pesquisadores. 
Os questionários foram entregues aos professores por bolsistas de iniciação científica, estudantes de Odontologia, treinados para esse fim, em horário previamente agendado, na própria instituição de ensino. Os estudantes aguardavam até que o professor respondesse ao questionário. Foram realizadas três tentativas de agendamento e entrega dos questionários, antes que o professor fosse considerado uma perda.

A variável dependente do estudo foi a presença de transtornos mentais comuns, avaliada pelo Questionário de Saúde Geral (QSG-12) validado no Brasil em uma amostra de bancários, de profissionais de saúde e desempregados (Borges e Argolo, 2002). O QSG-12 foi desenvolvido com o propósito de detectar transtornos mentais comuns ou não psicóticos e é um dos instrumentos mais utilizados para medir o bem-estar psicológico, principalmente, em estudos referentes à saúde do trabalhador (Borges e Argolo, 2002). O QSG-12 aponta as alterações na função mental que se apresentam como uma disfunção, deixando a pesquisa diagnóstica para uma abordagem psiquiátrica posterior (Goldberg et al., 1997; Gasparini, Barreto e Assunção, 2006). O instrumento contém 12 questões com quatro opções de respostas numa escala de concordância. A apuração das respostas seguiu o sistema de escore 0011, no qual se atribuiu zero às duas primeiras opções de respostas ou um para escolhas da terceira ou quarta categorias (Goldberg, 1997). Os escores totais variam de zero a 12 e o ponto de corte $3 / 4$ foi adotado para definir indivíduos sem ou com transtornos mentais comuns. $\mathrm{O}$ alpha de Cronbach do QSG-12 foi de 0,72.

Os estressores no trabalho foram avaliados adotando-se o modelo Esforço e Recompensa no Trabalho (Chor et al., 2008), que inclui dois componentes extrínsecos (esforço e recompensa) e um componente intrínseco (comprometimento excessivo). Foi utilizada a versão validada no Brasil (Chor et al., 2008). $\mathrm{O}$ instrumento contém 23 questões, seis referentes ao esforço, 11 à recompensa e seis ao comprometimento excessivo, cujas respostas variam no grau de concordância ou discordância, com escores que vão de um a cinco para esforço e recompensa e de um a quatro para comprometimento excessivo. Os escores totais de esforço podem variar de seis a trinta, e quanto maior o escore, maior o esforço envolvido e maior a percepção das demandas como estressoras. Na escala de recompensa, o escore total pode variar de 11 a 55, e quanto menor o escore, mais baixa será a percepção de recompensas no trabalho. Com relação ao comprometimento excessivo, os escores podem variar de quatro a 24 , e quanto maior o escore, maior é a vivência de excesso de comprometimento no trabalho (Siegrist et al., 2004). O alpha de Cronbach para as escalas de esforço, recompensa e comprometimento excessivo foram 0,76; 0,77 e 0,72, respectivamente.

As demais variáveis independentes investigadas foram:

- Informações sociodemográficas: sexo, faixa etária, situação conjugal (com companheiro e sem companheiro) e renda mensal em reais. 
- Formação profissional e condições de trabalho: anos de estudo, titulação (pós-doutorado, doutorado, mestrado, especialização e graduação), número de cursos que leciona na instituição, anos de docência, regime de trabalho, carga horária semanal, docência em outra instituição.

- Estilo de vida: hábito tabagista, consumo de bebidas alcoólicas e nível de atividade física.

Para os indivíduos que se declararam fumantes ou ex-fumantes, foi calculado o uso acumulado de cigarros na vida, considerando-se a intensidade diária e a duração do tabagismo na vida (Azevedo e Silva et al., 2009). No caso do consumo de bebidas alcoólicas, perguntou-se aos professores se consumiam ou já tinham consumido bebidas alcoólicas, sendo considerados não consumidores aqueles que relataram não beber atualmente ou nunca ter bebido na vida. O nível de atividade física foi medido pelo Questionário Internacional de Atividades Físicas (International Physical Activity Questionnaire, IPAQ), desenvolvido pela OMS, testado e validado no Brasil (Craig et al., 2003). O IPAQ foi analisado conforme orientações do Centro de Estudos do Laboratório de Aptidão Física de São Caetano do Sul e os professores foram classificados quanto ao nível de atividade física em muito ativo, ativo, irregularmente ativo e sedentário. Aqueles que realizaram atividades vigorosas, que exigem um grande esforço físico e fazem respirar muito mais forte do que o normal, por cinco ou mais dias na semana durante trinta minutos ou mais; ou ainda aqueles que realizaram atividades por três ou mais dias na semana durante vinte minutos contínuos juntamente com atividades moderadas foram classificados como muito ativos. As atividades moderadas são aquelas que necessitam de algum esforço físico e fazem respirar um pouco mais forte que o normal, e caminhada por, no mínimo, cinco dias na semana durante trinta minutos. Foram classificados como ativos aqueles que realizaram atividades físicas vigorosas por três dias na semana com sessões de no mínimo vinte minutos; atividade moderada ou caminhada, cinco ou mais dias por semana com duração mínima de trinta minutos/sessão; ou qualquer atividade somada: cinco dias por semana e cento e cinquenta minutos/semana. Os indivíduos classificados como irregularmente ativos praticavam atividades físicas por, pelo menos, dez minutos contínuos por semana, porém de maneira insuficiente para serem classificados como ativos. Já aqueles que não realizaram nenhuma atividade física por, pelo menos, dez minutos contínuos durante a semana foram classificados como sedentários (Centro de Estudos do Laboratório de Aptidão Física de São Caetano do Sul, 2008).

- Saúde geral: As variáveis de saúde geral avaliadas foram autoclassificação da saúde (ótima, boa, regular, ruim ou péssima), presença de doenças sis- 
têmicas diagnosticadas por um médico, uso de medicamentos prescritos por um médico e qualidade de vida. A qualidade de vida foi medida por meio do WHOQOL-bref, versão validada no Brasil (Fleck et al., 2000), que contém 24 perguntas referentes a quatro domínios (físico, psicológico, ambiente e social) e duas de ordem geral: "Como você avaliaria sua qualidade de vida?" e "Quão satisfeito você está com a sua saúde?". A análise do WHOQOL-bref seguiu as orientações da OMS (Harper e Power, 2011). Transformaram-se os escores em escalas de 0 a 100, e quanto maior o escore, melhor a qualidade de vida. Os questionários com $20 \%$ ou mais de perguntas sem respostas foram excluídos. O alpha de Cronbach do WHOQOL-bref foi 0,87.

Para a análise de dados empregou-se o programa SPSS ${ }^{\circledR}$ (Statistics Package for Social Science) versão 17.0 for Windows. A associação entre a presença de transtornos mentais e as variáveis independentes foi investigada por meio de análise bivariada e múltipla, empregando Análise de Regressão de Poisson. Incluíram-se no modelo múltiplo as variáveis associadas à presença de transtornos mentais com valor de $\mathrm{p}<0,20$ ou aquelas consideradas importantes para ajuste do modelo ou do ponto de vista teórico. Permaneceram no modelo final as variáveis associadas à presença de transtornos mentais com valor de $\mathrm{p}<0,05$. Os escores de qualidade de vida, de esforço, recompensa e comprometimento excessivo no trabalho foram incluídos no modelo como variáveis quantitativas. O alpha de Cronbach demonstrou a consistência interna das escalas empregadas.

Esta pesquisa faz parte do projeto aprovado pelo Comitê de Ética em Pesquisa da Funorte pelo protocolo $n^{\circ}$. 0392/09, e todos os participantes assinaram o termo de consentimento livre e esclarecido.

\section{Caracterização da amostra e prevalência de transtornos mentais comuns entre professores universitários}

Observaram-se transtornos mentais comuns em 19,5\% dos professores. Aproximadamente a metade dos professores era do sexo feminino $(53,1 \%)$ e $51,2 \%$ tinham entre 36 e 70 anos de idade. A maior parte tinha carga horária de, no máximo, vinte horas semanais $(68,2 \%)$ e trabalhava em outra instituição (70,7\%). Em relação aos hábitos comportamentais, a maioria consumia bebidas alcoólicas (74,6\%). A autopercepção da saúde geral como positiva (ótima/boa) foi relatada por $94,9 \%$ dos professores (Tabela 1). 
Tabela 1

Distribuição dos professores universitários segundo variáveis investigadas

\begin{tabular}{|c|c|c|}
\hline Variáveis & $\mathbf{N}$ & $\%$ \\
\hline \multicolumn{3}{|l|}{ SOCIODEMOGRÁFICAS } \\
\hline \multicolumn{3}{|l|}{$\operatorname{Sexo}(n=175)$} \\
\hline Feminino & 93 & 53,1 \\
\hline Masculino & 82 & 46,9 \\
\hline \multicolumn{3}{|l|}{ Faixa etária $(n=166)$} \\
\hline 26 a 35,5 anos & 81 & 48,8 \\
\hline 36 a 70 anos & 85 & 51,2 \\
\hline \multicolumn{3}{|l|}{ Situação conjugal ( $\mathrm{n}=173)$} \\
\hline Com companheiro & 120 & 69,4 \\
\hline Sem companheiro & 53 & 30,6 \\
\hline \multicolumn{3}{|l|}{ Renda mensal $(n=147)$} \\
\hline$R \$ 7.650,00$ a $R \$ 20.000,00$ & 36 & 24,5 \\
\hline $\mathrm{R} \$ 3.000,00$ a $R \$ 7.000,00$ & 72 & 49,0 \\
\hline $\mathrm{R} \$ 1.000,00$ a $R \$ 3.000,00$ & 39 & 26,5 \\
\hline \multicolumn{3}{|c|}{ FORMAÇÃO PROFISSIONAL E CONDIÇOEES DE TRABALHO } \\
\hline \multicolumn{3}{|l|}{ Anos de estudo $(n=165)$} \\
\hline 22 a 48 anos de estudo & 37 & 22,4 \\
\hline 19 a 21 anos de estudo & 80 & 48,5 \\
\hline 14 a 18 anos de estudo & 48 & 29,1 \\
\hline \multicolumn{3}{|l|}{ Titulação $(n=174)$} \\
\hline Pós-doutorado & 3 & 1,7 \\
\hline Doutorado & 3 & 1,7 \\
\hline Mestrado & 68 & 39,1 \\
\hline Especialização & 97 & 55,7 \\
\hline Graduação & 3 & 17,0 \\
\hline \multicolumn{3}{|l|}{ Anos de docência $(n=171)$} \\
\hline $2-5$ anos & 43 & 25,1 \\
\hline $6-12,5$ anos & 86 & 50,3 \\
\hline $13-40$ anos & 42 & 24,6 \\
\hline \multicolumn{3}{|c|}{ Regime de trabalho na instituição $(n=175)$} \\
\hline Integral & 20 & 11,4 \\
\hline Parcial + Horista & 155 & 88,6 \\
\hline \multicolumn{3}{|c|}{ Carga horária semanal na instituição $(n=170)$} \\
\hline 1 a 20 horas & 116 & 68,2 \\
\hline 21 horas ou mais & 54 & 31,8 \\
\hline \multicolumn{3}{|c|}{ Docência em outra instituiçăo ( $n=174)$} \\
\hline Não & 51 & 29,3 \\
\hline Sim & 123 & 70,7 \\
\hline \multicolumn{3}{|l|}{ ESTILO DE VIDA } \\
\hline \multicolumn{3}{|l|}{ Hábito tabagista $(n=175)$} \\
\hline Não fumante & 158 & 90,3 \\
\hline Fumante + ex-fumante & 17 & 9,7 \\
\hline
\end{tabular}


Continuação - Tabela 1

Distribuição dos professores universitários segundo variáveis investigadas

\begin{tabular}{|c|c|c|}
\hline Variáveis & $\mathrm{N}$ & $\%$ \\
\hline \multicolumn{3}{|l|}{ Consumo de bebidas alcóolicas ( $n=173$ ) } \\
\hline Não & 93 & 25,4 \\
\hline Sim & 82 & 74,6 \\
\hline \multicolumn{3}{|l|}{ Nível de atividade física $(n=173)$} \\
\hline Ativo + Muito ativo & 81 & 57,2 \\
\hline Sedentário + Irregularmente ativo & 85 & 42,8 \\
\hline \multicolumn{3}{|l|}{ SAÚDE GERAL } \\
\hline \multicolumn{3}{|l|}{ Autoclassificação da saúde ( $n=175)$} \\
\hline Ótima & 56 & 32,0 \\
\hline Boa & 110 & 62,9 \\
\hline Regular & 9 & 5,1 \\
\hline \multicolumn{3}{|c|}{ Doenças sistêmicas diagnostica das por um médico $(n=171)$} \\
\hline Sem doença sistêmica & 120 & 70,2 \\
\hline Com doença sistêmica & 51 & 29,8 \\
\hline \multicolumn{3}{|c|}{ Uso de medicamentos prescritos por um médico $(n=168)$} \\
\hline Não & 116 & 69,0 \\
\hline Sim & 52 & 31.0 \\
\hline
\end{tabular}

Fonte: Os autores.

\section{Fatores associados aos transtornos mentais comuns entre professores universitários}

$\mathrm{Na}$ análise bivariada, as seguintes variáveis foram associadas à presença de transtornos mentais comuns, com valor de $\mathrm{p}<0,20$ e foram selecionadas para o modelo múltiplo: faixa etária, regime de trabalho, carga horária semanal, escores de esforço no trabalho, escores de comprometimento excessivo, autoclassificação da saúde e escores dos domínios físico e psicológico da qualidade de vida (Tabela 2). Incluíram-se ainda no modelo as variáveis sexo, tempo de docência, situação conjugal e escores de recompensa no trabalho. 
Tabela 2

Resultado da análise bivariada entre presença de transtornos mentais comuns e variáveis independentes investigadas

\begin{tabular}{|c|c|c|c|c|c|c|}
\hline \multirow[t]{2}{*}{ Variáveis } & \multicolumn{2}{|c|}{$\begin{array}{l}\text { Com transtorno } \\
\text { mental comum }\end{array}$} & \multicolumn{2}{|c|}{$\begin{array}{l}\text { Sem transtorno } \\
\text { mental comum }\end{array}$} & \multirow[t]{2}{*}{$\begin{array}{l}\text { RP bruta* } \\
\text { (IC 95\%) }\end{array}$} & \multirow[t]{2}{*}{$\begin{array}{l}\text { RP bruta* } \\
\text { (IC 95\%) }\end{array}$} \\
\hline & $\mathrm{N}$ & $\%$ & $\mathrm{~N}$ & $\%$ & & \\
\hline \multicolumn{7}{|l|}{ SOCIODEMOGRÁFICAS } \\
\hline \multicolumn{7}{|l|}{ Sexo } \\
\hline Feminino & 21 & 23,1 & 70 & 76,9 & 1 & 0,22 \\
\hline Masculino & 12 & 15,4 & 66 & 84,6 & $0,67(0,35-1,27)$ & \\
\hline \multicolumn{7}{|l|}{ Faixa etária } \\
\hline 26 a 35,5 anos & 19 & 24,7 & 58 & 75,3 & 1 & 0,16 \\
\hline 36 a 70 anos & 13 & 15,7 & 70 & 84,3 & $0,64(0,34-1,20)$ & \\
\hline \multicolumn{7}{|l|}{ Estado civil } \\
\hline Com companheiro & 20 & 17,2 & 96 & 82,8 & 1 & 0,21 \\
\hline Sem companheiro & 13 & 25,5 & 38 & 74,5 & $1,48(0,80-2,74)$ & \\
\hline \multicolumn{7}{|l|}{ Renda mensal } \\
\hline $\mathrm{R} \$ 7.650,00$ a $\mathrm{R} \$ 20.000,00$ & 7 & 20,6 & 27 & 79,4 & 1 & \\
\hline $\mathrm{R} \$ 3.000,00$ a $R \$ 7.000,00$ & 13 & 18,6 & 57 & 81,4 & $0,90(0,40-2,05)$ & 0,81 \\
\hline $\mathrm{R} \$ 1.000,00$ a $\mathrm{R} \$ 3.000,00$ & 11 & 28,2 & 28 & 71,8 & $1,37(0,6-3,14)$ & 0,46 \\
\hline \multicolumn{7}{|c|}{ FORMAÇÃO PROFISSIONAL E CONDIÇŐES DE TRABALHO } \\
\hline \multicolumn{7}{|l|}{ Anos de estudo } \\
\hline 22 a 48 anos & 7 & 20,0 & 28 & 80,0 & 1 & \\
\hline 19 a 21 anos & 16 & 21,1 & 60 & 78,9 & $1,05(0,48-2,33)$ & 0,90 \\
\hline 14 a 18 anos & 8 & 16,7 & 40 & 83,3 & $0,83(0,33-2,08)$ & 0,70 \\
\hline \multicolumn{7}{|l|}{ Titulação } \\
\hline Mestrado + Doutorado + Pós-doutorado & 15 & 22,1 & 53 & 77,9 & 1 & \\
\hline Graduação + Especialização & 18 & 18,6 & 79 & 81,4 & $0,84(0,46-1,55)$ & 0,58 \\
\hline \multicolumn{7}{|l|}{ Anos de docência } \\
\hline 2 a 5 anos & 10 & 23,8 & 32 & 76,6 & 1 & \\
\hline 6 a 12,5 anos & 17 & 20,0 & 68 & 80,0 & $0,84(0,42-1,67)$ & 0,62 \\
\hline 13 a 40 anos & 5 & 13,2 & 33 & 86,8 & $0,55(0,21-1,47)$ & 0,24 \\
\hline \multicolumn{7}{|l|}{ Regime de trabalho na instituição } \\
\hline Integral & 6 & 31,6 & 13 & 68,4 & 1 & \\
\hline Parcial + Horista & 27 & 18,0 & 123 & 82,0 & $0,57(0,27-1,20)$ & 0,14 \\
\hline \multicolumn{7}{|l|}{ Carga horária semanal na instituição } \\
\hline 1 a 20 horas & 18 & 16,2 & 93 & 83,8 & 1 & \\
\hline 21 horas ou mais & 13 & 24,5 & 40 & 75,5 & $1,51(0,80-2,85)$ & 0,20 \\
\hline \multicolumn{7}{|l|}{ Docência em outra instituição } \\
\hline Não & 11 & 22.0 & 39 & 78,0 & 1 & \\
\hline Sim & 22 & 18,6 & 96 & 81,4 & $0,85(0,45-1,61)$ & 0,61 \\
\hline Esforço no trabalho & & & & & $1,10(1,03-1,18)$ & 0,00 \\
\hline Recompensa no trabalho & & & & & $1,03(0,98-1,07)$ & 0,25 \\
\hline Comprometimento excessivo no trabalho & & & & & $1,18(1,02-1,36)$ & 0,02 \\
\hline \multicolumn{7}{|l|}{ ESTILO DE VIDA } \\
\hline \multicolumn{7}{|l|}{ Hábito tabagista } \\
\hline Não é tabagista & 31 & 20,4 & 121 & 79,6 & 1 & \\
\hline Fumante + Ex-fumante & 2 & 11,8 & 15 & 88,2 & $0,58(0,15-2,20)$ & 0,42 \\
\hline
\end{tabular}


Continuação - Tabela 2

Resultado da análise bivariada entre presença de transtornos mentais comuns e variáveis independentes investigadas

\begin{tabular}{|c|c|c|c|c|c|c|}
\hline \multirow[t]{2}{*}{ Variáveis } & \multicolumn{2}{|c|}{$\begin{array}{l}\text { Com transtorno } \\
\text { mental comum }\end{array}$} & \multicolumn{2}{|c|}{$\begin{array}{l}\text { Sem transtorno } \\
\text { mental comum }\end{array}$} & \multirow[t]{2}{*}{$\begin{array}{l}\text { RP bruta* } \\
\text { (IC 95\%) }\end{array}$} & \multirow[t]{2}{*}{$\begin{array}{l}\text { RP bruta* } \\
\text { (IC 95\%) }\end{array}$} \\
\hline & & & $\mathrm{N}$ & $\%$ & & \\
\hline \multicolumn{7}{|l|}{ Consumo de bebidas alcóolicas } \\
\hline Não & 7 & 16,3 & 36 & 83,7 & 1 & \\
\hline Sim & 25 & 20,2 & 99 & 79,8 & $1,24(0,58-2,66)$ & 0,58 \\
\hline \multicolumn{7}{|l|}{ Atividade física } \\
\hline Ativo + Muito ativo & 16 & 16,3 & 82 & 83,7 & 1 & \\
\hline Sedentário + Irregularmente ativo & 17 & 24,6 & 52 & 75,4 & $1,51(0,82-2,78)$ & 0,19 \\
\hline \multicolumn{7}{|l|}{ SAÚDE GERAL } \\
\hline \multicolumn{7}{|l|}{ Autoclassificação da saúde } \\
\hline Ótima & 6 & 10,9 & 49 & 89,1 & 1 & \\
\hline Boa + Regular & 27 & 23,8 & 87 & 76,3 & $0,46(0,20-1,05)$ & 0,06 \\
\hline \multicolumn{7}{|c|}{ Doenças sistêmicas diagnosticadas por um médico } \\
\hline Sem doença sistêmica & 23 & 20,0 & 92 & 80,0 & $0,80(0,38-1,67)$ & 0,55 \\
\hline Com doença sistêmica & 8 & 16,0 & 42 & 84,0 & 1 & \\
\hline \multicolumn{7}{|l|}{ Uso de medicamentos prescritos por um médico } \\
\hline Não faz uso de medicamentos & 21 & 18,9 & 90 & 81,1 & 1 & \\
\hline Faz uso de medicamentos & 10 & 19,6 & 41 & 80,4 & $1,04(0,53-2,04)$ & 0,92 \\
\hline Qualidade de vida geral & & & & & $1,00(0,99-1,02)$ & 0,79 \\
\hline Domínio físico da qualidade de vida & & & & & $0,96(0,94-0,98)$ & 0,00 \\
\hline Domínio social da qualidade de vida & & & & & $0,99(0,97-1,02)$ & 0,56 \\
\hline Domínio psicológico da qualidade de vida & & & & & $0,98(0,94-1,01)$ & 0,13 \\
\hline Domínio ambiente da qualidade de vida & & & & & $1,01(0,98-1,04)$ & 0,59 \\
\hline
\end{tabular}

Fonte: Os autores.

*RP = Razão de prevalência.

$\mathrm{Na}$ análise múltipla, o esforço no trabalho e o domínio físico da qualidade de vida foram significativamente associados à presença de transtornos mentais comuns, independentemente das outras variáveis incluídas no modelo. A prevalência de transtornos mentais comuns foi maior entre professores com maiores escores de esforço no trabalho $(\mathrm{RP}=1,07$, IC $95 \%=1,01-1,14)$ e diminuiu com o aumento dos escores do domínio físico da qualidade de vida $(\mathrm{RP}=0,95$, IC $95 \%=0,94-0,97)$ (Tabela 3). O aumento de uma unidade nos escores de esforço correspondeu a um aumento de $7 \%$ na prevalência de transtornos mentais comuns. 
Tabela 3

Modelo de regressão de Poisson final dos fatores associados à presença de transtornos mentais comuns

\begin{tabular}{lcc}
\hline Variáveis & $\begin{array}{c}\text { RP ajustada } \\
\text { (IC 95\%) }\end{array}$ & Valor de $\mathbf{p}$ \\
\hline Domínio físico da qualidade de vida & $0,95(0,94-0,97)$ & 0,000 \\
Esforço no trabalho & $1,07(1,01-1,14)$ & 0,043 \\
\hline
\end{tabular}

Fonte: Os autores.

Entre os professores considerados com estresse pela escala de esforço no trabalho (aqueles que optaram por uma das três opções de resposta às questões de esforço: concordo, um pouco estressado ou concordo, estressado ou concordo, muito estressado), a frequência dos considerados com transtornos mentais comuns foi significativamente maior para a grande parte deles (Tabela 4).

Tabela 4

Distribuição da frequência de transtorno mental comum entre os professores com respostas positivas (com estresse) para cada uma das questões da escala de esforço

\begin{tabular}{|c|c|c|c|}
\hline Questões da escala de esforço & $\begin{array}{c}\text { Com transtorno } \\
\text { mental }\end{array}$ & $\begin{array}{l}\text { Sem transtorno } \\
\text { mental }\end{array}$ & Valor de $\mathrm{p}^{* *}$ \\
\hline $\begin{array}{l}\text { Eu me sinto constantemente pressionado para fazer muito } \\
\text { trabalho em pouco tempo }\end{array}$ & 15,2 & 2,2 & 0,00 \\
\hline Constantemente interrompem e atrapalham meu trabalho & 12,1 & 4,4 & 0,09 \\
\hline Eu tenho muitas responsabilidades no meu trabalho & 18,2 & 2,2 & 0,00 \\
\hline $\begin{array}{l}\text { Eu sou pressionado com frequência a trabalhar além do } \\
\text { horário }\end{array}$ & 9,1 & 2,2 & 0,05 \\
\hline Meu trabalho exige esforço físico & 9,1 & 0,7 & 0,00 \\
\hline $\begin{array}{l}\text { Nos últimos anos, meu trabalho tem se tornado cada vez } \\
\text { mais exigente }\end{array}$ & 12,1 & 4,4 & 0,09 \\
\hline
\end{tabular}

Fonte: Os autores.

${ }^{*}$ A frequência de transtorno mental comum foi obtida entre aqueles com respostas positivas para as questões de esforço.

**Valor de $\mathrm{p}$ - Resultado do teste qui-quadrado. 


\section{Discussão: uma reflexão sobre a prevalência e os fatores associados aos transtornos mentais comuns entre professores universitários}

Esse estudo demonstrou que a prevalência de transtornos mentais comuns entre professores de nível superior da área da saúde de uma instituição do norte de Minas Gerais foi de 19,5\%, sendo maior naqueles com maior escore de esforço no trabalho e que apresentaram pior qualidade de vida no domínio físico. A prevalência observada nessa população foi maior do que a prevalência de depressão de 15,6\% , observada na população em geral, diagnosticada com o uso da Classificação Internacional de Doenças - $10^{a}$ revisão (CID-10) (Vorcaro et al., 2001).

Tal resultado é preocupante, ainda mais considerando que, aproximadamente, metade dos professores tinha menos de 35 anos de idade. A elevada prevalência de transtornos mentais em uma população jovem pode ser um indicativo de um processo de desgaste acelerado que promove uma série de alterações negativas na saúde dos professores (Vorcaro et al., 2001). Os distúrbios psíquicos raramente são fatais, porém são comuns, duradouros ou transitórios e recorrentes, podendo causar o afastamento dos professores de suas atividades (Porto et al., 2006).

A prevalência de transtorno mental na população estudada foi inferior à encontrada entre professores da rede municipal de ensino fundamental da região nordeste de Belo Horizonte (50,3\%) (Gasparini, Barreto e Assunção, 2006) e entre professores do ensino infantil e municipal da rede pública municipal e de escolas particulares em Vitória da Conquista, Bahia (44\%) (Porto et al., 2006).

No primeiro estudo, foram utilizados o mesmo instrumento e o mesmo ponto de corte para definir professores com e sem transtorno mental. Porém, nos professores do ensino fundamental de Belo Horizonte, a prevalência de transtorno mental foi associada à experiência anterior com violência no trabalho e falta de estrutura física, como iluminação precária. Esses fatores estressores podem ter menos impacto na saúde dos professores universitários, podendo explicar a menor prevalência observada no presente estudo. Além disso, os autores afirmaram que a alta prevalência tenha sido influenciada pela época da coleta de dados, final do último semestre, momento de acúmulo de atividades.

No segundo estudo, foi utilizado o SRQ-20, sendo observada associação com exigências no trabalho. O SRQ-20 é um questionário para identificação de transtornos mentais comuns (TMC) composto por vinte questões cujas respostas são do tipo sim/não. Os escores obtidos estão relacionados com a probabilidade de presença de transtorno não-psicótico, variando de zero (nenhuma probabilidade) a vinte (extrema probabilidade). Para uma pessoa ser considerada com transtorno mental, utiliza-se a pontuação de sete ou mais respostas afirmativas. 
A prevalência observada no presente estudo (19,5\%) foi similar à encontrada por Vedovato e Monteiro (2008) em pesquisa com professores estaduais de ensino fundamental ou médio de cidades paulistas $(20,9 \%)$. Outro estudo nacional entre professores de ciências de uma escola pública do Rio Grande do Sul mostrou que $83 \%$ dos professores relataram sentirem-se nervosos, tensos ou preocupados.

Um estudo entre professores universitários no Reino Unido, utilizando a Hospital Anxiety and Depression Scale, encontrou uma prevalência de $31,6 \%$ e 7,8\% para a ansiedade e a depressão, respectivamente (Mark e Smith, 2012). Embora as metodologias e os instrumentos adotados para avaliação dos distúrbios psíquicos tenham sido diferentes, verifica-se, nas pesquisas consultadas, que os professores apresentam uma considerável prevalência dessas alterações. A frequência de transtorno mental observada no presente estudo foi semelhante à encontrada entre profissionais que lidam com saúde mental: médicos, psicólogos, assistentes sociais, enfermeiro, auxiliares de enfermagem, terapeutas ocupacionais, fonoaudiólogo, antropólogo e dentistas (15,8\%) (Marco et al., 2008). Apesar de o ambiente de trabalho diferir, as categorias profissionais avaliadas foram semelhantes, o que pode justificar resultados concordantes.

$\mathrm{O}$ adoecimento mental entre professores pode estar relacionado não somente à intensificação do trabalho docente, ao elevado volume de trabalho, à precariedade das condições existentes, mas também à diversidade e à complexidade das questões presentes na sala de aula e, ainda, a uma expectativa social de excelência (Glina et al., 2001; Delcor et al., 2004; Gasparini, Barreto e Assunção, 2006).

No presente estudo não se comparou a prevalência de transtornos mentais entre professores de universidades públicas e privadas. Contudo, em estudos anteriores, argumenta-se que, em universidades privadas, os docentes estão mais sujeitos a adoecer. Segundo D'Antola (1992), os professores de escolas privadas estão submetidos a processos e organização do trabalho distintos dos existentes nas escolas públicas, o que pode ocasionar frequentes distorções salariais, inseguranças e incertezas.

Estudo qualitativo entre docentes de universidades privadas brasileiras observou que o reconhecimento social é um dos elementos na construção da identidade dos docentes das instituições particulares.

(...) desencantamento e o sofrimento são bastante comuns entre esses docentes no confronto do dia a dia das instituições e da gestão educacional que operam mediante critérios de mercado que impactam o trabalho desses docentes; e é nesse sentido que suas identidades vêm sendo construídas, permeadas pela angústia em torno da questão da demissão associada ao fracasso em relação à carreira ou o exercício profissional (Siqueira, 2006, p. 811). 
Apesar do aumento, em termos absolutos, da demanda dos cursos de graduação e da expansão da oferta de vagas nas instituições privadas, tem ocorrido, em termos relativos, uma retração na procura dessas instituições, o que pode ser comprovado pela redução na relação candidatos/vaga nesse segmento. O docente da universidade privada passa a temer a demissão por não existirem novas turmas no curso em que leciona (Schwartzman, 2001). Assim, a insegurança no trabalho pode implicar pessimismo, baixa autoestima, ansiedade e tristeza. Em pesquisa anterior demonstrou-se que docentes da universidade privada apresentaram autoestima mais baixa do que aqueles que lecionavam nas universidades públicas (Terra, Marziale e Robazzi, 2013).

Vários autores constataram associação entre condições de trabalho, estresse ocupacional e presença de distúrbios mentais entre professores de níveis secundário e médio (Gasparini, Barreto e Assunção, 2006; Porto et al., 2006; Vedovato e Monteiro, 2008), e um prejuízo da qualidade de vida (Rocha e Fernandes, 2008; Yang et al., 2009). No presente estudo, observou-se que o aumento nos escores de esforço no trabalho foi acompanhado do aumento na prevalência de professores com transtornos mentais. Esperava-se que maior recompensa fosse inversamente associada à prevalência de transtornos mentais, pois o modelo Esforço-Recompensa e Comprometimento excessivo baseia-se na reciprocidade de intercâmbio na vida profissional. De acordo com o modelo, uma pessoa com maior necessidade de controle responde de maneira inflexível às situações de trabalho que exigem muito esforço e oferecem baixa recompensa, o que acaba por acarretar estresse e predispor ao adoecimento (Van Vegchel et al., 2005; Siegrist, 2005).

A associação observada neste estudo evidenciou que professores com distintos níveis do componente esforço apresentaram diferentes prevalências de transtornos mentais, significando que condições do trabalho docente, tais como demandas e obrigações, podem predispor o trabalhador a respostas desfavoráveis. O componente esforço incluiu questões que avaliaram a sensação de sentir-se pressionado devido a carga de trabalho, nível de responsabilidade no trabalho, frequência com que é pressionado a trabalhar fora do horário, aumento da exigência do trabalho com o tempo. Tal resultado vai ao encontro das inquietações dos docentes na Universidade Federal de Santa Catarina, que relataram que as condições de trabalho estão se tornando cada vez mais insalubres, competitivas e estressantes (Fontana e Pinheiro, 2010). Possui também relação com a observação de que, entre trabalhadores universitários no Reino Unido, o componente esforço apresentou correlação positiva com os escores de ansiedade $(r=0,476)$ e depressão $(r=0,479)$ (Mark e Smith, 2012).

A prevalência de transtornos mentais foi menor nos professores com maiores escores de qualidade de vida no domínio físico. A presença de 
transtorno mental representa a manifestação de sintomas depressivos e de ansiedade. Tal quadro pode comprometer o desempenho físico diário do professor. O domínio físico da qualidade de vida avalia o quanto a dor impede as atividades, quanto se necessita de tratamento para levar a vida diária, se o indivíduo apresenta energia suficiente para o seu dia a dia, se está satisfeito com a capacidade para se locomover, com o desempenho das atividades diárias e com a capacidade para o trabalho, condições que podem estar comprometidas quando o indivíduo encontra-se em um estado de depressão. Em estudos anteriores, evidenciou-se a associação entre transtorno mental e prejuízo da qualidade de vida (Jardim, Barreto e Assunção, 2007; Rocha e Fernandes, 2008; Marco et al., 2008).

Para avaliação de transtorno mental comum, elegeu-se o QSG-12, por ser um instrumento curto e indicado para "conhecer o nível de saúde mental das populações em risco" (Jardim, Barreto e Assunção, 2007) e investigar o bem-estar psicológico em estudos ocupacionais (Borges e Argolo, 2002). Estudos brasileiros mostraram validade da sua estrutura unifatorial em amostra de população geral, entre bancários e profissionais de saúde empregados e desempregados (Borges e Argolo, 2002) e adequada consistência interna (alpha de Cronbach $=0,80$ ), também observada na amostra de professores deste estudo.

Embora alguns pesquisadores tenham discutido solução bifatorial, os resultados são ainda inconclusivos (Borges e Argolo, 2002; Gouveia et al., 2003). Não se identificaram estudos que avaliassem a reprodutibilidade desse instrumento. Quanto à escala de esforço, recompensa e comprometimento excessivo no trabalho, a versão utilizada no presente estudo apresentou propriedades psicométricas adequadas em estudo de Chor et al., 2008: as estimativas de confiabilidade (coeficiente de correlação intraclasse) das três dimensões da escala, esforço, recompensa e comprometimento excessivo foram de 0,76, 0,86, e 0,78, respectivamente. Estimativas de consistência interna (alpha de Cronbach para essas mesmas dimensões foram de 0,68, 0,78 , e 0,78 . A estrutura de fatores, obtida por meio de análise fatorial exploratória, mostrou-se bastante consistente com as bases teóricas do modelo (Chor et al., 2008). As estimativas de consistência interna satisfatórias foram também observadas na amostra de professores incluída no presente estudo. O WHOQOL-bref apresentou características satisfatórias de consistência interna, validade discriminante, validade de critério, validade concorrente e fidedignidade teste-reteste (Fleck et al., 2000).

Apesar de a taxa de resposta obtida ser considerada boa (Babbie, 2005), pode ter ocorrido uma subestimativa da prevalência de distúrbios mentais e de níveis de estresse no trabalho devido à perda, pois os não respondentes podem ser um subgrupo com maior sobrecarga de trabalho. Outra limitação deste estudo é que os dados são baseados em avaliações subjetivas. Alguns 
autores sugerem que usar o mesmo método para avaliar as variáveis independentes e dependentes leva a uma correlação inflacionada. Essa situação pode ser explicada pela afetividade negativa, uma característica da personalidade que predispõe a emoções negativas e pessimismo. Assim, essas pessoas tendem a considerar sua vida como pior, seu trabalho mais estressante e sua qualidade de vida mais afetada do que outros sem afetividade negativa (Arial e Wild, 2011). A amostra deste estudo não permite generalizar os resultados para os professores de outras instituições, públicas ou privadas. Novas pesquisas, com amostras representativas, devem ser realizadas, incluindo professores de universidades públicas e privadas a fim de comparar o efeito dos dois diferentes contextos na saúde desses trabalhadores.

Conclui-se que há uma prevalência considerável de transtornos mentais comuns entre professores universitários da área da saúde de uma instituição privada, o que corrobora estudos na literatura. Há maior prevalência de transtornos mentais comuns nos professores que se esforçam mais no trabalho (com maior frequência de estressores) e com pior qualidade de vida no domínio físico.

\section{Colaboradores}

Raquel Conceição Ferreira e Andréa Maria Eleutério de Barros Lima Martins participaram da análise dos dados, interpretação dos resultados, redação e revisão final do artigo. Alessandra Pastore da Silveira, Maria Aparecida Barbosa de Sá, Sara de Barros Lima Feres e João Gabriel Silva Souza participaram da coleta de dados, análise de dados e redação do artigo. Não há conflitos de interesse de qualquer natureza. 
Resumen Los profesores universitarios están expuestos a un aumento de tensión en el trabajo dada la fragmentación de su actividad y las responsabilidades exigidas, sin que, en muchas situaciones, tengan las condiciones necesarias para responder adecuadamente. Dicha situación puede representar condiciones estresoras, aumentando el riesgo de trastornos mentales. Se investigó la asociación entre trastornos mentales comunes y estresores en el trabajo, entre profesores de nueve cursos de salud en una universidad particular en Minas Gerais, Brasil. La variable dependiente fue la presencia de trastornos mentales, medida por el Cuestionario de Salud General 12. Los estresores en el trabajo se evaluaron por el modelo Esfuerzo-Recompensa y Compromiso excesivo. Las demás variables fueron: sociodemográficas, historia ocupacional, comportamentales y referentes a la salud general. Los datos fueron sometidos a análisis descriptivo, análisis bivariante y regresión de Poisson. Participaron 175 profesores (80,0\%) y el 19,5\% presentó trastornos mentales comunes. La prevalencia de estos trastornos fue mayor entre profesores con mayor esfuerzo en el trabajo $(\mathrm{RP}=1,8$; IC95\%=1,01-3,46) y menor en aquellos con mayor calidad de vida en el dominio físico $(\mathrm{RP}=0,95$, IC95\%=0,93-0,97). Se concluye que existe una prevalencia considerable de trastornos mentales comunes entre profesores universitários, y es mayor en aquellos que se esfuerzan más en el trabajo y con peor calidad de vida en el dominio físico.

Palabras clave salud mental; docentes; calidad de vida; salud del trabajador; agotamiento profesional.

\section{Notas}

1 Departamento de Odontologia Social e Preventiva, Faculdade de Odontologia, Universidade Federal de Minas Gerais, Belo Horizonte, Minas Gerais, Brasil.

$<$ ferreira_rc@hotmail.com>

Correspondência: Av. Antônio Carlos, 6.627, Bairro Pampulha, CEP 31270-901, Belo Horizonte, Minas Gerais, Brasil.

2 Faculdades Unidas do Norte de Minas, Montes Claros, Minas Gerais, Brasil. $<$ alessandrapastore150@hotmail.com>

3 Faculdades Unidas do Norte de Minas, Montes Claros, Minas Gerais, Brasil. <cidab.d.s@hotmail.com>

4 Faculdades Unidas do Norte de Minas, Montes Claros, Minas Gerais, Brasil. $<$ sara_feress@hotmail.com>

5 Faculdade de Odontologia de Piracicaba, Universidade Estadual de Campinas, Campinas, São Paulo, Brasil.

<jgabriel.ssouza@yahoo.com.br>

6 Departamento de Odontologia, Centro de Ciências Biológicas e da Saúde, Universidade Estadual de Montes Claros, Montes Claros, Minas Gerais, Brasil.

$<$ martins.andreamebl@gmail.com> 


\section{Referências}

ABOUSERIE, Reda. Stress, coping strategies and job satisfaction in university academic staff. Educational Psychology, Hong Kong, v. 16, n. 1, p. 49-56, 1996.

ARIAL, Marc; WILD, Pascal. Effort, reward and self-reported mental health: a simulation study on negative affectivity bias. BMC Medical Research Methodology, London, v. 11, p. 121, 2011.

BABBIE, Earl. Métodos de pesquisa de Survey. Belo Horizonte: Editora UFMG, 2005. 519 p.

BORGES, Livia O.; ARGOLO, João C. T. Adaptação e validação de uma escala de bem-estar psicológico para uso em estudos ocupacionais. Avaliação psicológica, Porto Alegre, v. 1, n. 1, p. 17-27, 2002.

BRASIL. Ministério da Saúde. Doenças relacionadas ao trabalho: manual de procedimentos para os serviços de saúde. Brasília: MS, 2001.

BRUM, Liliani M. et al. Qualidade de vida dos professores da área de ciências em escola pública no Rio Grande do Sul. Trabalho, Educação e Saúde, v. 10, n. 1, p. 125-145, 2012.

CARLOTTO, Mari S.; PALAZZO, Lilian S Síndrome de Burnout e fatores associados: um estudo epidemiológico com professores. Cadernos de Saúde Pública, Rio de Janeiro, v. 22, n. 5, p. 1.017-1.026, 2006.

CARLOTTO, Mari S. Síndrome de Burnout e satisfação no trabalho: um estudo com professores universitários. In: PEREIRA, Ana Maria T. B. Burnout: Quando o trabalho ameaça o bem-estar do trabalhador. São Paulo: Casa do Psicólogo, 2010. p. 187-212.

CENTRO DE ESTUDOS DO LABORATÓRIO DE APTIDÃO FÍSICA DE SÃO CAETANO DO SUL (CELAFISCS). Questionário Internacional de Atividade Física: versão curta. 2008. Disponível em: <www.portalagita.org.br/ uploads/agita_saopaulo/arquivos/IPAQ classificacao.pdf> Acesso: 14 abr. 2011.
CHOR, Dóra et al. The Brazilian version of the effort reward imbalance questionnaire to assess job stress. Cadernos de Saúde Pública, Rio de Janeiro, v. 24, n. 1, p. 219-224, 2008.

CRAIG, Cora L. et al. International physical activity questionnaire: 12-country reliability and validity. Medicine and Science in Sports and Exercises, Indianápolis, v. 35, n. 8, p. 1.381-1.395, 2003.

D'ANTOLA, Arlete A. A prática docente na universidade. São Paulo: EPU; 1992. 187 p.

DELCOR, Nuria S. et al. Condições de trabalho e saúde dos professores da rede particular de ensino de Vitória da Conquista, Bahia, Brasil. Cadernos de Saúde Pública, Rio de Janeiro, v. 20, n. 1, p. 187-196, 2004.

FERNANDES, Marcos H.; ROCHA, Vera M. Impact of the psychosocial aspects of work on the quality of life of teachers. Revista Brasileira de Psiquiatria, São Paulo, v. 31, n. 1, p. 15-20, 2009.

FLECK, Marcelo P. A. et al. Aplicação da versão em português do instrumento abreviado de avaliação da qualidade de vida "WHOQOL-bref". Revista de Saúde Pública, São Paulo, v. 34, n. 2, p. 178-183, 2000.

FONTANA, Rosane T.; PINHEIRO, Débora A. Condições de saúde autorreferidas de professores de uma universidade regional. Revista Gaúcha de Enfermagem, Porto Alegre, v. 31, n. 2, p. 270-276, 2010.

GASPARINI, Sandra M.; BARRETO, Sandhi M.; ASSUNÇÃO, Ada A. Prevalência de transtornos mentais comuns em professores da rede municipal de Belo Horizonte, Minas Gerais, Brasil. Cadernos de Saúde Pública, Rio de Janeiro, v. 22, n. 12, p. 2.679-2.691, 2006.

GILLESPIE, Nicole A. et al. Occupational stress in universities: staff perceptions of the causes, consequences and moderators of stress. Work \& Stress, London, v. 15, n. 1, p. 53-72, 2001. 
GLINA, Débora M. R. et al. Saúde mental e trabalho: uma reflexão sobre o nexo com o trabalho e o diagnóstico, com base na prática. Cadernos de Saúde Pública, Rio de Janeiro, v. 17, n. 3, p. 607-616, 2001.

GOLDBERG, David P. et al. The validity of two versions of the GHQ in the WHO study of mental illness in general health care. Psychological Medicine, Cambridge, v. 27, n. 1, p. 191-197, 1997.

GONÇALVES, Daniel M.; KAPCZINSKI, Flávio P. Transtorno mental, indicadores demográficos e satisfação com a vida. Revista de Saúde Pública, São Paulo, v. 42, n. 6, p. 1.060-1.066, 2008.

GOUVEIA, Valdiney V. et al. A utilização do QSG-12 na população geral: estudo de sua validade de construto. Psicologia: Teoria e Pesquisa, Brasília, v. 19, n. 3, p. 241-248, 2003.

HARPER, Alison; POWER, Mick. Steps for checking and cleaning data and computing domain scores for the WHOQOL-BREF. Disponível em: <www.ufrgs.br/psiq/Sintaxe. pdf $>$. Acesso em: 15 jun. 2011.

JACQUES, Maria G. O nexo causal em saúde/doença mental no trabalho: uma demanda para a psicologia. Psicologia \& Sociedade, Porto alegre, v. 19, p. 112-119, 2007.

JARDIM, Renata; BARRETO, Sandhi M.; ASSUNÇÃO, Ada A. Condições de trabalho, qualidade de vida e disfonia entre docentes. Cadernos de Saúde Pública, Rio de Janeiro, v. 23, n. 10, p. 2.439-2.461, 2007.

LEUNG, Tat-Wing; SIU, Oi-Ling; SPECTOR, Paul E. Faculty stressors, job satisfaction, and psychological distress among university teachers in Hong Kong: The role of locus of control. International Journal of Stress Management, Washington, v. 7, n. 2, p. 121-138, 2000.

LUDERMIR, Ana B.; MELO FILHO, Djalma A. Condições de vida e estrutura ocupacional associadas a transtornos mentais co- muns. Revista de Saúde Pública, São Paulo, v. 36, n. 2, p. 213-221, 2002.

MARCO, Patrícia F. et al. O impacto do trabalho em saúde mental: transtornos psiquiátricos menores, qualidade de vida e satisfação profissional. Jornal Brasileiro de Psiquiatria, Rio de Janeiro, v. 57, n. 3, p. 78-183, 2008.

MARK, George; SMITH, Andrew. Effects of occupational stress, job characteristics, coping, and attributional style on the mental health and job satisfaction of university employees Anxiety, Stress and Coping, London, v. 25, n. 1, p. 63-78, 2012.

ORGANIZAÇÃO MUNDIAL DE SAÚDE. Organização Pan-Americana de Saúde. Saúde mental: nova concepção, nova esperança. Genebra: OMS, 2001. (Relatório sobre a Saúde no Mundo 2001).

PORTO, Lauro A. et al. Associação entre distúrbios psíquicos e aspectos psicossociais do trabalho de professores. Revista de Saúde Pública, São Paulo, v. 40, n. 5, p. 818826, 2006.

ROCHA, Vera M.; FERNANDES, Marcos H. Qualidade de vida de professores do ensino fundamental: uma perspectiva para a promoção da saúde do trabalhador. Jornal Brasileiro de Psiquiatria, Rio de Janeiro, v. 57, n. 1, p. 23-27, 2008.

SCHWARTZMAN, Simon A. Revolução Silenciosa do Ensino Superior. In: DURHAM, Eunice. R.; SAMPAIO, Helena. O Ensino Superior em transformação. São Paulo: Núcleo de Pesquisas sobre Ensino Superior, 2001. p. 12-35.

SIEGRIST, Johannes et al. The measurement of effort-reward imbalance at work: European comparisons. Social Science \& Medicine, Amsterdam, v. 58, n. 8, p. 1.4831.499, 2004.

SIEGRIST, Johannes. Social reciprocity and health: new scientific evidence and policy implications. Psychoneuroendocrinology, Amsterdam, v. 30, n. 10, p. 1.033-1.038, 2005. 
SILVA, Gulnar A. et al. Tabagismo e escolaridade no Brasil, 2006. Revista de Saúde Pública, São Paulo, v. 43, p. 48-56, 2009. (Suplemento 2).

SIQUEIRA, Tania C. A. O trabalho docente nas instituições de ensino superior privado em Brasília. Saúde e Sociedade, São Paulo, v. $21, \mathrm{n}, 13,810-811,2006$.

SOUSA, Ivone F.; MENDONÇA, Helenides. Burnout em professores universitários: impacto de percepções de justiça e comprometimento afetivo. Psicologia: Teoria e Pesquisa, Brasília, v. 25, n. 4, p. 499-508, 2009.

TERRA, Fabio S.; MARZIALE, Maria H. P.; ROBAZZI, Maria L. C. C. Avaliação da autoestima em docentes de enfermagem de universidades pública e privada. Revista Latino-Americana de Enfermagem, Ribeirão Preto, v. 21, feb. 2013. (Número especial). Disponível em: <www.scielo.br/scielo.php?script= sci_arttext\&pid=S0104-11692013000700010\& $\operatorname{lng}=$ en\&nrm=iso $>$. Acesso em: 17 jun. 2014.
VAN VEGCHEL, Natasja et al. Reviewing the effort-reward imbalance model: Drawing up the balance of 45 empirical studies. Social Science \& Medicine, Amsterdam, v. 60, n. 5, p. 1.117-1.131, 2005.

VEDOVATO, Tatiana G.; MONTEIRO, Maria I. Perfil sociodemográfico e condições de saúde e trabalho dos professores de nove escolas estaduais paulistas. Revista da Escola de Enfermagem da USP, São Paulo, v. 42, n. 2, p. 290-297, 2008.

VORCARO, Claudia. M. R. et al. Unexpected high prevalence of 1-month depression in a small Brazilian community: the Bambuí study. Acta Psychiatrica Scandinavica, Malden, v. 104, n. 4, p. 257-263, 2001.

YANG, Xiaolu et al. Relationship between quality of life and occupational stress among teachers. Public Health, Amsterdam, v. 123, n. 11, p. 750-755, 2009.

Recebido em 13/11/2013

Aprovado em 03/08/2014 